\title{
Jejunal Obstruction, CTCAE
}

National Cancer Institute

\section{Source}

National Cancer Institute. Jejunal Obstruction, CT CAE. NCI Thesaurus. Code C57829.

A disorder characterized by blockage of the normal flow of the intestinal contents in the jejunum. 